\title{
Detection of a dense clump in a filament interacting with W51e2 ${ }^{\star}$
}

\author{
B. Mookerjea ${ }^{1}$, C. Vastel $^{2,3}$, G. E. Hassel ${ }^{4}$, M. Gerin ${ }^{5}$, J. Pety ${ }^{6,5}$, P. F. Goldsmith ${ }^{7}$, J. H. Black ${ }^{8}$, T. Giesen ${ }^{9}$, \\ T. Harrison ${ }^{4}$, C. M. Persson ${ }^{8}$, and J. Stutzki ${ }^{10}$ \\ 1 Tata Institute of Fundamental Research, Homi Bhabha Road, 400005 Mumbai, India \\ e-mail: bhaswati@tifr.res.in \\ 2 Université de Toulouse, UPS-OMP, IRAP, Toulouse, France \\ 3 CNRS, IRAP, 9 Av. colonel Roche, BP 44346, 31028 Toulouse Cedex 4, France \\ 4 Department of Physics and Astronomy, Siena College, Loudonville NY 12211, USA \\ 5 LERMA, Observatoire de Paris ENS, and UMR8112 du CNRS, 24 rue Lhomond, 75231 Paris Cedex 05, France \\ 6 IRAM, 300 rue de la Piscine, 38406 St. Martin d'Heres, France \\ 7 Jet Propulsion Laboratory, California Institute of Technology, 4800 Oak Grove Boulevard, Pasadena, CA 91109, USA \\ 8 Onsala Space Observatory, Chalmers University of Technology, 43992 Onsala, Sweden \\ 9 Institute of Physics, University of Kassel, 34132 Kassel, Germany \\ 10 I. Physikalisches Institut, University of Cologne, 50937 Cologne, Germany
}

Received 26 November 2013 / Accepted 19 March 2014

\section{ABSTRACT}

\begin{abstract}
In the framework of the Herschel/PRISMAS guaranteed time key program, the line of sight to the distant ultracompact $\mathrm{H}$ II region W51e2 has been observed using several selected molecular species. Most of the detected absorption features are not associated with the background high-mass star-forming region and probe the diffuse matter along the line of sight. We present here the detection of an additional narrow absorption feature at $\sim 70 \mathrm{~km} \mathrm{~s}^{-1}$ in the observed spectra of $\mathrm{HDO}, \mathrm{NH}_{3}$ and $\mathrm{C}_{3}$. The $70 \mathrm{~km} \mathrm{~s}^{-1}$ feature is not uniquely identifiable with the dynamic components (the main cloud and the large-scale foreground filament) so-far identified toward this region. The narrow absorption feature is similar to the one found toward low-mass protostars, which is characteristic of the presence of a cold external envelope. The far-infrared spectroscopic data were combined with existing ground-based observations of ${ }^{12} \mathrm{CO},{ }^{13} \mathrm{CO}, \mathrm{CCH}, \mathrm{CN}$, and $\mathrm{C}_{3} \mathrm{H}_{2}$ to characterize the $70 \mathrm{~km} \mathrm{~s}^{-1}$ component. Using a non-LTE analysis of multiple transitions of $\mathrm{NH}_{3}$ and $\mathrm{CN}$, we estimated the density $\left(n\left(\mathrm{H}_{2}\right) \sim(1-5) \times 10^{5} \mathrm{~cm}^{-3}\right)$ and temperature $(10-30 \mathrm{~K})$ for this narrow feature. We used a gasgrain warm-up based chemical model with physical parameters derived from the $\mathrm{NH}_{3}$ data to explain the observed abundances of the different chemical species. We propose that the $70 \mathrm{~km} \mathrm{~s}^{-1}$ narrow feature arises in a dense and cold clump that probably undergoes collapse to form a low-mass protostar, formed on the trailing side of the high-velocity filament, which is thought to be interacting with the W51 main cloud. While the fortuitous coincidence of the dense clump along the line of sight with the continuum-bright W51e2 compact $\mathrm{H}$ II region has contributed to its nondetection in the continuum images, this same attribute makes it an appropriate source for absorption studies and in particular for ice studies of star-forming regions.
\end{abstract}

Key words. ISM: molecules - submillimeter: ISM - ISM: lines and bands - line: formation - line: identification - molecular data

\section{Introduction}

The high-sensitivity large-scale CO maps of giant molecular clouds provided the first evidence for the filamentary nature of the interstellar medium (ISM; Ungerechts \& Thaddeus 1987; Bally et al. 1987). The far-infrared all-sky IRAS survey and several mid-infrared surveys (ISO, Spitzer/MIPS) revealed the ubiquitous filamentary structure of both the dense and diffuse ISM (Low et al. 1984). Most recently, the unprecedented sensitivity and large-scale mapping capabilities of Herschel/SPIRE have revealed a large network of parsec-scale filaments in Galactic molecular clouds and have suggested an intimate connection between the filamentary structure of the ISM and the formation of dense cloud cores (André et al. 2010; Molinari et al. 2010a). Here we present evidence of the detection of a dense clump possibly formed by the interaction of an extended foreground filament with the giant molecular cloud W51.

* Based on data acquired with Herschel and IRAM observatories. Herschel is an ESA space observatory with science instruments provided by European-led Principal Investigator consortia and with important participation from NASA.
Located at a distance of $5.41_{-0.28}^{+0.31} \mathrm{kpc}$ (Sato et al. 2010), W51 is a radio source with a complicated morphology in which many compact sources are superposed on extended diffuse emission (Bieging 1975). The line of sight to W51 intersects the Sagittarius spiral arm nearly tangentially $\left(1=49^{\circ}\right)$, which means that sources over a $\sim 5 \mathrm{kpc}$ range of distances are superimposed on the line of sight. Based on the ${ }^{12} \mathrm{CO}$ and ${ }^{13} \mathrm{CO} 1-0$ line emission of these sources, Carpenter \& Sanders (1998) divided the molecular gas associated with the W51 H II region into two subgroups: a giant molecular cloud $\left(1.2 \times 10^{6} M_{\odot}\right)$ at $\sim 61 \mathrm{~km} \mathrm{~s}^{-1}$, and an elongated $(22 \times 136 \mathrm{pc})$ molecular cloud akin to a filamentary structure $\left(1.9 \times 10^{5} M_{\odot}\right)$ at $68 \mathrm{~km} \mathrm{~s}^{-1}$. While the brightest radio source at $6 \mathrm{~cm}(\mathrm{G} 49.5-0.4)$ is spatially and kinematically coincident with the W51 giant molecular cloud, the G49.2-0.3, G49.1-0.4, G49.0-0.3, and G48.9-0.3 radio sources seem to be associated with the $68 \mathrm{~km} \mathrm{~s}^{-1}$ cloud. Carpenter \& Sanders (1998) speculated that the massive star formation activity in this region resulted from a collision between the W51 giant molecular cloud and the high-velocity $\left(68 \mathrm{~km} \mathrm{~s}^{-1}\right)$ cloud. G49.5-0.4 is the brightest source in the W51 main region. The continuum emission from the ultracompact $\mathrm{H}$ II region was 


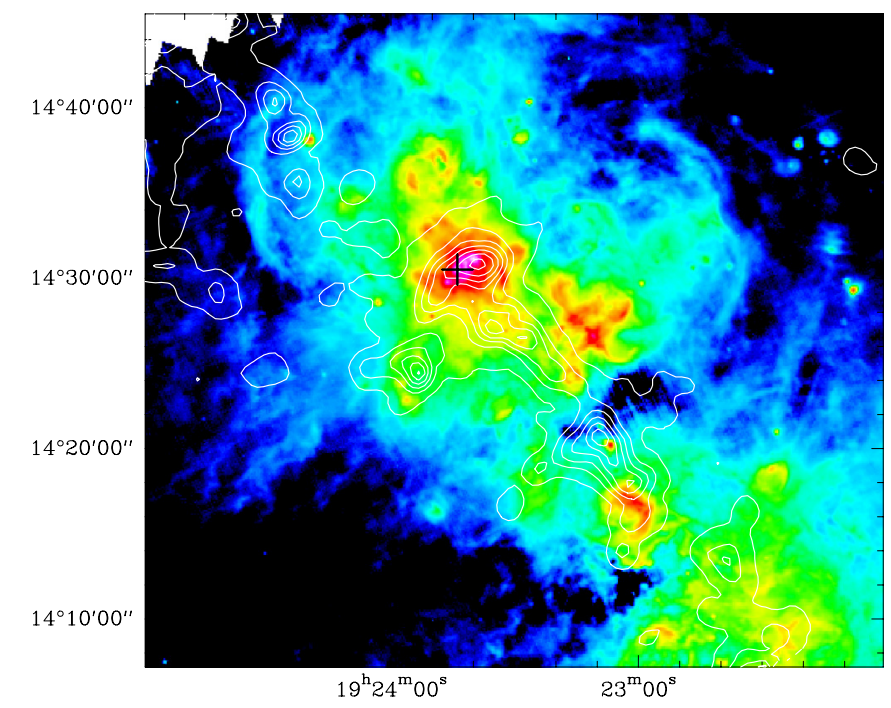

Fig. 1. Color image shows $70 \mu \mathrm{m}$ continuum emission observed with PACS. The white contours represent ${ }^{13} \mathrm{CO}(1-0)$ emission integrated between 68.9 and $70.3 \mathrm{~km} \mathrm{~s}^{-1}$ corresponding to the "filament", observed using FCRAO as part of the Galactic Ring Survey. The black cross shows the position of the HIFI observations.

collectively named W51e before it was resolved into compact components, labeled as W51e1 to W51e8 (Scott 1978; Gaume et al. 1993; Mehringer 1994; Zhang \& Ho 1997). Figure 1 shows the $70 \mu \mathrm{m}$ continuum emission as observed with PACS by Hi-Gal (Molinari et al. 2010b) in color, along with contours of ${ }^{13} \mathrm{CO}(1-0)$ emission integrated between 68.9 and $70.3 \mathrm{~km} \mathrm{~s}^{-1}$ to locate the GMC and the filament ${ }^{1}$. The continuum emission appears to be better correlated with the filament, possibly suggesting star formation activity in the filament.

As part of the Herschel key program "PRobing InterStellar Molecules with Absorption line Studies (PRISMAS) we have observed several selected spectral lines in absorption toward the source W51e2 to study the foreground material along the line of sight using HIFI. These observations have for the first time detected in absorption a component at $70 \mathrm{~km} \mathrm{~s}^{-1}$ (a velocity even higher than the velocity of the $68 \mathrm{~km} \mathrm{~s}^{-1}$ filament) along with the $57 \mathrm{~km} \mathrm{~s}^{-1}$ source velocity component, in $\mathrm{C}_{3}, \mathrm{HDO}$, and $\mathrm{NH}_{3}$. The observation of $\mathrm{C}_{3}$ was motivated by the importance of small carbon chains for the chemistry of stellar and interstellar environments either as building blocks for the formation of long carbon chain molecules, or as are products of photofragmentation of larger species such as PAHs (Radi et al. 1988; Pety et al. 2005). $\mathrm{C}_{3}$ has been identified with Herschel/HIFI for the first time in the warm envelopes of star-forming hot cores like W31C, W49N and DR21(OH) (Mookerjea et al. 2010, 2012). Within the PRISMAS program, the ground-state HDO transition at $894 \mathrm{GHz}$ has only been detected at the velocity of the H II regions. Based on observations of high-mass star-forming regions (Jacq et al. 1990; Pardo et al. 2001; van der Tak et al. 2006; Persson et al. 2007; Bergin et al. 2010) and low-mass protostars (Liu et al. 2011; Coutens et al. 2012, 2013a), the $\mathrm{HDO} / \mathrm{H}_{2} \mathrm{O}$ ratio remains lower than the $\mathrm{D} / \mathrm{H}$ ratio derived for other deuterated molecules observed in the same sources although it is clearly higher than the cosmic $\mathrm{D} / \mathrm{H}$ abundance. This low deuterium

\footnotetext{
The PACS data shown in Fig. 1 are a Level 2 data product produced by the standard data reduction pipeline for PACS-SPIRE parallel mode observations as obtained from the Herschel Science Archive. The ${ }^{13} \mathrm{CO}$ data have taken from the Galactic Ring Survey done by FCRAO available online at http://www.bu.edu/galacticring/
}

enrichment of water could provide an important constraint to the astrochemical models to explain the chemical processes involved in the formation of water. Ammonia $\left(\mathrm{NH}_{3}\right)$ is a key species in the nitrogen chemistry and a valuable diagnostic because its complex energy level structure covers a very broad range of critical densities and temperatures. It has been observed throughout the ISM ever since its detection in 1968 (Cheung et al. 1968) but mainly in its inversion lines at $\mathrm{cm}$ wavelengths. With Herschel it became possible to observe ground-state rotational transitions with high critical densities at $\mathrm{THz}$ frequencies of both ortho and para symmetries, for instance, in cold envelopes of protostars (Hily-Blant et al. 2010), in diffuse/translucent interstellar gas (Persson et al. 2012), and hot cores (Neill et al. 2013).

We have combined the HIFI observations with IRAM observations of $\mathrm{CCH}, \mathrm{c}-\mathrm{C}_{3} \mathrm{H}_{2}$ and $\mathrm{CN}$ in all of which the $70 \mathrm{~km} \mathrm{~s}^{-1}$ feature is detected in absorption. We have used local thermodynamic equilibrium (LTE) and non-LTE models to derive the physical parameters of the dense clump at $70 \mathrm{~km} \mathrm{~s}^{-1}$ and its relation to the W51 molecular cloud. We also present a gas-grain, warm-up chemical model that consistently explains the abundances of all the species observed in the clump. This paper is organized as follows: Sects. 2 and 3 describe the Herschel and IRAM observations, respectively. Section 4 describes the complex velocity structure observed along the sightline to W51e2. Section 5 discusses the column and volume densities derived from all the tracers that detect the $70 \mathrm{~km} \mathrm{~s}^{-1}$ clump. In Sect. 6 we compare the observed abundances of the various chemical species with a grain warm-up based chemical model. Section 7 discusses various derived properties of the $70 \mathrm{~km} \mathrm{~s}^{-1}$ clump and presents a possible formation scenario for the clump.

\section{Observations with Herschel/HIFI}

In the framework of the Herschel key program PRISMAS (P. I. M. Gerin), we observed the line of sight toward the compact $\mathrm{H}$ II region W51e2, whose coordinates are $\alpha(J 2000)=$ $19^{\mathrm{h}} 23^{\mathrm{m}} 43^{\mathrm{s}} .90 \delta(J 2000)=14^{\circ} 30^{\prime} 30^{\prime} .^{\prime} 5$. The observations were performed in the pointed dual-beam switch (DBS) mode using the HIFI instrument (de Graauw et al. 2010; Roelfsema et al. 2012) onboard Herschel (Pilbratt et al. 2010). The DBS reference positions were situated approximately $3^{\prime}$ east and west of the source. The HIFI Wide Band Spectrometer (WBS) was used with optimization of the continuum, providing a spectral resolution of $1.1 \mathrm{MHz}$ over an instantaneous bandwidth of $4 \times 1 \mathrm{GHz}$. To separate the lines of interest from the lines in the other sideband, which could possibly contaminate our observations, we observed the same transition with three different LO settings. This method is necessary in such chemically rich regions to ensure genuine identification of spectral lines. The data were processed using the standard HIFI pipeline up to level 2 with the ESA-supported package HIPE 8.0 (Ott 2010) and were then exported as FITS files into CLASS/GILDAS format ${ }^{2}$ for subsequent data reduction. Both polarizations were averaged to reduce the noise in the final spectrum. The baselines obtained and subtracted are well fitted by straight lines over the frequency range of the whole band. The single sideband continuum temperature derived from the polynomial fit at the line frequency was added back to the spectra.

We followed up the detection of the $70 \mathrm{~km} \mathrm{~s}^{-1}$ velocity component in $\mathrm{C}_{3}$ by searching for the feature in different available

\footnotetext{
See http://wWw.iram. fr/IRAMFR/GILDAS for more information about the GILDAS software.
} 
Table 1. Spectroscopic parameters for the observed spectral lines.

\begin{tabular}{|c|c|c|c|c|c|c|c|}
\hline Species & Telescope & Transition & $\begin{array}{c}\text { Frequency } \\
\text { GHz }\end{array}$ & $\begin{array}{r}E_{\text {low }} \\
\mathrm{K}\end{array}$ & $\begin{array}{l}A_{\mathrm{ul}} \\
\mathrm{s}^{-1}\end{array}$ & Beam size & Reference \\
\hline \multirow[t]{4}{*}{$\mathrm{C}_{3},(J, v)$} & Herschel/HIFI & $(9,1)-(10,0) \mathrm{P}(10)$ & 1654.081660 & 68.1 & $2.38 \times 10^{-3}$ & $12^{\prime \prime}$ & $a$ \\
\hline & Herschel/HIFI & $(3,1)-(4,0) \mathrm{P}(4)$ & 1787.890568 & 12.4 & $2.72 \times 10^{-3}$ & $12^{\prime \prime}$ & $a$ \\
\hline & Herschel/HIFI & $(2,1)-(2,0) \mathrm{Q}(2)$ & 1890.558057 & 3.7 & $7.51 \times 10^{-3}$ & $10^{\prime \prime}$ & $a$ \\
\hline & Herschel/HIFI & $(4,1)-(4,0) \mathrm{Q}(4)$ & 1896.706558 & 12.4 & $7.58 \times 10^{-3}$ & $10^{\prime \prime}$ & $a$ \\
\hline $\mathrm{c}-\mathrm{C}_{3} \mathrm{H}_{2},\left(J_{K_{\mathrm{a}}}, K_{\mathrm{c}}\right)$ & IRAM-30 m & $2_{1,2}-1_{0,1}$ & 85.338893 & 2.35 & $2.32 \times 10^{-5}$ & 28.13 & $\mathrm{CDMS}^{b}$ \\
\hline \multirow[t]{10}{*}{$\mathrm{CN}$} & IRAM-30 m & $1,1 / 2,1 / 2-0,1 / 2,1 / 2$ & 113.123370 & 0.0 & $1.29 \times 10^{-6}$ & $22^{\prime \prime}$ & CDMS \\
\hline & IRAM-30 m & $1,1 / 2,1 / 2-0,1 / 2,3 / 2$ & 113.144157 & 0.0 & $1.05 \times 10^{-5}$ & $22^{\prime \prime}$ & CDMS \\
\hline & IRAM-30 m & $1,1 / 2,3 / 2-0,1 / 2,1 / 2$ & 113.170492 & 0.0 & $5.15 \times 10^{-6}$ & $22^{\prime \prime}$ & CDMS \\
\hline & IRAM-30 m & $1,1 / 2,3 / 2-0,1 / 2,3 / 2$ & 113.191279 & 0.0 & $6.68 \times 10^{-6}$ & $22^{\prime \prime}$ & CDMS \\
\hline & IRAM-30 m & $2,5 / 2,5 / 2-1,3 / 2,1 / 2$ & 226.874191 & 5.5 & $9.62 \times 10^{-5}$ & $11^{\prime \prime}$ & CDMS \\
\hline & IRAM-30 m & $2,5 / 2,7 / 2-1,3 / 2,5 / 2$ & 226.874781 & 5.5 & $1.14 \times 10^{-4}$ & $11^{\prime \prime}$ & CDMS \\
\hline & IRAM-30 m & $2,5 / 2,3 / 2-1,3 / 2,1 / 2$ & 226.875896 & 5.5 & $8.59 \times 10^{-5}$ & $11^{\prime \prime}$ & CDMS \\
\hline & IRAM-30 m & $2,5 / 2,3 / 2-1,3 / 2,3 / 2$ & 226.887420 & 5.5 & $2.73 \times 10^{-5}$ & $11^{\prime \prime}$ & CDMS \\
\hline & IRAM-30 m & $2,5 / 2,5 / 2-1,3 / 2,5 / 2$ & 226.892128 & 5.5 & $1.81 \times 10^{-5}$ & $11^{\prime \prime}$ & CDMS \\
\hline & IRAM-30 m & $2,5 / 2,3 / 2-1,3 / 2,5 / 2$ & 226.905357 & 5.5 & $1.13 \times 10^{-6}$ & $11^{\prime \prime}$ & CDMS \\
\hline $\mathrm{CCH}, \mathrm{N}_{J, F}$ & IRAM-PdBI & $1_{3 / 2,1}-0_{1 / 2,0}$ & 87.328585 & 0.0 & $1.27 \times 10^{-6}$ & $5{ }^{\prime \prime} 6 \times 44^{\prime \prime} 6$ & CDMS \\
\hline para- $\mathrm{H}_{2}^{18} \mathrm{O}$ & Herschel/HIFI & $1_{11}-0_{00}$ & 1101.698257 & 0.0 & $1.79 \times 10^{-2}$ & 19.2 & $\mathrm{JPL}^{c}$ \\
\hline \multirow{2}{*}{ ortho- $\mathrm{NH}_{3}$} & Herschel/HIFI & $1_{0}^{-}-0_{0}^{+}$ & 572.498160 & 0.0 & $1.58 \times 10^{-3}$ & $37 . .5$ & JPL \\
\hline & Herschel/HIFI & $2_{0}^{+}-1_{0}^{-}$ & 1214.852942 & 27.5 & $1.81 \times 10^{-2}$ & $17 . .5$ & JPL \\
\hline \multirow[t]{3}{*}{ para- $\mathrm{NH}_{3}$} & Herschel/HIFI & $2_{1}^{-}-1_{1}^{+}$ & 1168.452394 & 23.3 & $1.21 \times 10^{-2}$ & $17 . .5$ & JPL \\
\hline & Herschel/HIFI & $2_{1}^{+}-1_{1}^{-}$ & 1215.245714 & 22.2 & $1.36 \times 10^{-2}$ & $17 . .5$ & JPL \\
\hline & Herschel/HIFI & $3_{2}^{+}-2_{2}^{-}$ & 1763.823186 & 64.5 & $3.30 \times 10^{-2}$ & $12^{\prime \prime}$ & JPL \\
\hline HDO & Herschel/HIFI & $1_{1,1}^{2}-0_{0,0}^{2}$ & 893.638666 & 0.0 & $8.35 \times 10^{-6}$ & $244^{\prime \prime} 1$ & JPL \\
\hline
\end{tabular}

References. ${ }^{(a)}$ Mookerjea et al. (2010); ${ }^{(b)}$ Müller et al. (2005); and ${ }^{(c)}$ Pickett et al. (1998).

transitions of $\mathrm{HDO}, \mathrm{NH}_{3}, \mathrm{CCH}, \mathrm{c}-\mathrm{C}_{3} \mathrm{H}_{2}$, and $\mathrm{CN}$. Among these chosen molecules, $\mathrm{c}-\mathrm{C}_{3} \mathrm{H}_{2}$ and $\mathrm{CCH}$ are chemically related to $\mathrm{C}_{3}$ since they are the by-products of the proposed formation route of $\mathrm{C}_{3}$ via warm-up of the $\mathrm{CH}_{4}$ mantle of the grains, which essentially refers to a temperature of $30 \mathrm{~K}$ and higher (Hassel et al. 2011). In contrast, $\mathrm{NH}_{3}$ and $\mathrm{HDO}$ are tracers of cold $(20 \mathrm{~K})$ and dense molecular gas. The abundance of $\mathrm{CN}$ is typically found to be low in hot cores, and higher in regions of intense $\mathrm{UV}$ fields such as the interfaces of $\mathrm{H}$ II regions and molecular clouds.

\subsection{Linear carbon chain, $C_{3}$}

We observed four transitions of the $v_{2}$ fundamental band, $P(4)$, $Q(2), Q(4)$, and $P(10)$ of triatomic carbon using the bands $7 \mathrm{a}, 7 \mathrm{~b}$, and $6 \mathrm{~b}$ of the HIFI receiver (Müller et al. 2005; Mookerjea et al. 2010). All spectroscopic information regarding the observed $C_{3}$ lines is presented in Table 1 . The observations were carried out on October 29, 2010 and April 22 and 23, 2011. Further details of the observations and data reduction are similar to the analysis of the W31C and W49N sources presented in Mookerjea et al. (2010). All spectra were smoothed to resolutions of between $\sim 0.16$ to $0.18 \mathrm{~km} \mathrm{~s}^{-1}$ and the rms noise level for the spectra ranges between 0.03 to $0.04 \mathrm{~K}$ (Fig. 2).

\section{2. $\mathrm{NH}_{3}$}

We observed the ortho- $\mathrm{NH}_{3} 2_{0}^{+}-1_{0}^{-}$and $1_{0}^{-}-0_{0}^{+}$ground-state transitions at 1214.8 (band 5a) and $572.5 \mathrm{GHz}$ (band $1 \mathrm{~b}$ ) as well as the para- $\mathrm{NH}_{3} 2_{1}^{+}-1_{1}^{-}$ground transition at $1215.2 \mathrm{GHz}$ (band 5a) (Yu et al. 2010). The beam size is $\sim 37^{\prime \prime}$ for the low frequency transition and $17.5^{\prime \prime}$ for the higher frequencies transitions. The forward efficiency is 0.96 and the main beam efficiencies are 0.76 (at $572 \mathrm{GHz}$ ) and 0.64 (at $1215 \mathrm{GHz}$ ). The PRISMAS

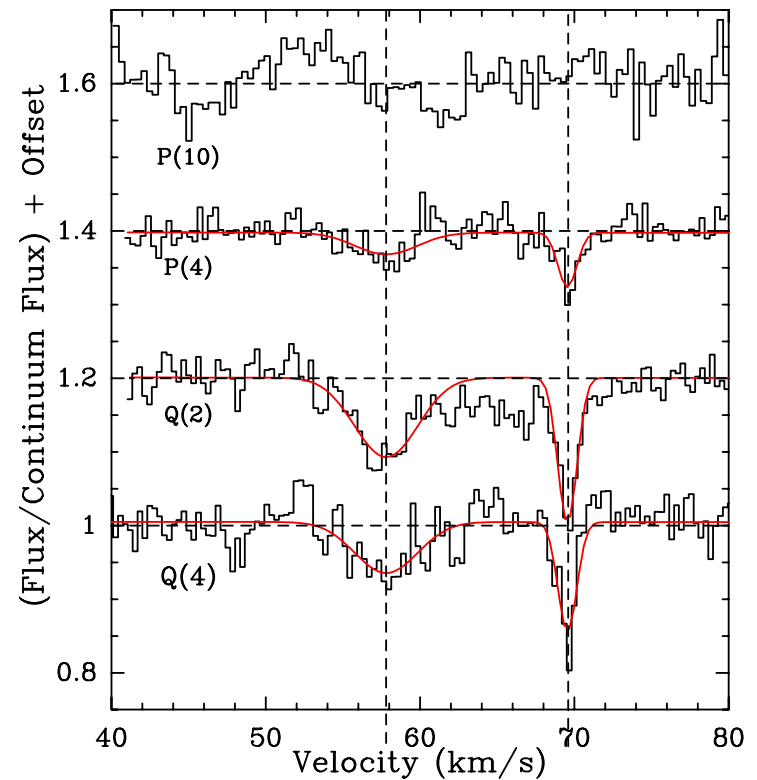

Fig. 2. HIFI observations of the $\mathrm{C}_{3}$ lines. The observed spectra (in black) are corrected for the DSB continuum and normalized to the SSB continuum level. Results of simultaneous fitting of $P(4), Q(2)$ and $Q(4)$ are shown in red. The observed spectrum of $P(10)$ is also shown to illustrate the nondetection.

observations were carried out on October 29, 2010 and April 22, 2011. Additionally, we used the para- $\mathrm{NH}_{3} 2_{1}^{-}-1_{1}^{+}$transition at $1168.45 \mathrm{GHz}$ and $3_{2}^{+}-2_{2}^{-}$transition at $1763.82 \mathrm{GHz}$ observations obtained within the OT1 programme on absorption studies of N-hydrides. These observations were performed on April 18 and 21, 2012. Spectroscopic and observational details for all transitions are summarized in Table 1. 
Table 2. Observation parameters for $\mathrm{CCH}$ with $\mathrm{PdBI}$.

\begin{tabular}{ccccccccc}
\hline \hline & \multicolumn{3}{c}{ Phase center } & & Number of fields & Field-of-view & Obs. date \\
W51 & $\alpha_{2000}=19^{\mathrm{h}} 23^{\mathrm{m}} 43.40^{\mathrm{s}}$ & $\delta_{2000}=14^{\circ} 30^{\prime} 34.8^{\prime \prime}$ & 2 & & $72^{\prime \prime} \times 58^{\prime \prime}$ & 7-Sep.-2007 \\
\hline \multirow{3}{*}{ Configuration } & Beam & PA & Resol & Water & $T_{\text {sys }}$ & On-source & Noise $^{a}$ \\
& & arcsec & $\circ$ & $\mathrm{km} \mathrm{s}^{-1}$ & $\mathrm{~mm}$ & $\mathrm{~K}$ & hours & mJy/Beam \\
W51 & $\mathrm{D}$ & $5.61 \times 4.63$ & 132.6 & 0.27 & 4 & 100 & 1.5 & 9 \\
\hline
\end{tabular}

Notes. ${ }^{(a)}$ The noise values quoted here are those at the mosaic center (mosaic noise is inhomogeneous because of primary beam correction; it steeply increases at the mosaic edges).

\section{3. $H D O$}

We observed one of the HDO fundamental transitions $\left(1_{1,1}-0_{0,0}\right)$ at $893.6 \mathrm{GHz}$ (using the spectroscopic catalog JPL: Pickett et al. 1998 ) in band 3b. The beam is about $24^{\prime \prime} 1$ and the forward efficiency and the main beam efficiency are 0.96 and 0.74 , respectively. The observations were carried out on October 27 , 2010. Spectroscopic and observational details are summarized in Table 1.

\section{Ground-based IRAM observations}

\section{1. $\mathrm{CCH}$ with $\mathrm{PdBI}$}

Plateau de Bure Interferometer (PdBI) observations dedicated to the PRISMAS project were carried out with six antennas in September 2007. The $4 \mathrm{GHz}$ instantaneous IF-bandwidth allowed us to simultaneously observe $\mathrm{CCH}$ (plus six other lines) at $3 \mathrm{~mm}$ using seven different $40 \mathrm{MHz}$-wide correlator windows. One additional $320 \mathrm{MHz}$-wide correlator window was used to simultaneously measure the $3 \mathrm{~mm}$ continuum. The $\mathrm{CCH}$ line was detected in emission and absorption (spectroscopic details in Table 1).

We observed W51e2 in track-sharing mode in the D configuration (baseline lengths from 24 to $97 \mathrm{~m}$ ). A two-fields Nyquist-sampled mosaic was needed to cover the W51e 2 continuum emission. The D configuration was observed for about $7 \mathrm{~h}$ of telescope time. This leads to on-source integration time of useful data of $1.5 \mathrm{~h}$ for W51e 2 after removing the bad data. The rms amplitude noise of the bandpass calibration was $0.5 \%$. The rms phase noise were between 7 and $18^{\circ}$, which introduced position errors of $<0$ '.25. The typical resolution is 5'. 1 and the primary beam at the frequency of the $\mathrm{CCH}$ transition at $87.328585 \mathrm{GHz}$ is $56^{\prime \prime} 6$. Table 2 presents the relevant parameters for these observations.

The data were reduced with the GILDAS software supported at IRAM (Pety 2005). Standard calibration methods using nearby calibrators were applied. The calibrators used for the absolute flux calibration are B1749+096 (5.2 Jy) and B1923+210 (1.4 Jy), respectively. The W51e2 data were processed using natural weighting to obtain the optimal signal-to-noise ratio. The line emission extension exceeds one third of the primary beam, implying the need for short-spacings from a single-dish telescope. However, we are interested here in the absorption lines in front of the continuum source, which is compact enough to avoid the need for short-spacings. The data were deconvolved using the Högbom CLEAN algorithm with a support defined around the continuum source to guide the search of the CLEAN components.

\section{2. $\mathrm{CN}$ and $\mathrm{c}-\mathrm{C}_{3} \mathrm{H}_{2}$ with IRAM $30 \mathrm{~m}$}

The CN $J=1-0$ transition was observed at the IRAM-30 m telescope at Pico Veleta (Spain) in December 2006 in wobblerswitching mode and detected through its $F=1 / 2-1 / 2,1 / 2-3 / 2$,
$3 / 2-1 / 2,3 / 2-3 / 2$ hyperfine components (see Table 1 ). All the details about these observations can be found in Godard et al. (2010). The $\mathrm{CN} J=2-1$ transition was also observed in parallel with $J=1-0$ in its $F=5 / 2-3 / 2,7 / 2-5 / 2,3 / 2-1 / 2,3 / 2-3 / 2$, $5 / 2-5 / 2$ and $3 / 2-5 / 2$ hyperfine components (see Table 1 ), although this is not reported in Godard et al. (2010). The halfpower beam width is about $22^{\prime \prime}$ at $113 \mathrm{GHz}$ compared with the $11^{\prime \prime}$ value at $227 \mathrm{GHz}$.

The $\mathrm{c}-\mathrm{C}_{3} \mathrm{H}_{2} 2_{1,2}-1_{0,1}$ transition at $85.3 \mathrm{GHz}$ was observed in December 2006 (Gerin et al. 2011). The spectroscopic details are quoted in Table 1.

\section{New velocity component along the W51e2 sightline}

To determine the spatial distribution of gas in the W51e 2 region, the velocity components originating in the local gas need to be separated from those produced by gas along the line of sight to the background continuum source. Fortunately, this source was observed in detail using several transitions of atomic and molecular species. For example, $\mathrm{H} 109 \alpha$ observations toward W51e2 showed that the average velocity of the ionized gas is $\simeq 56.8 \mathrm{~km} \mathrm{~s}^{-1}$ (Wilson et al. 1970). Koo (1997) used H I observations toward W51e 2 to identify two sets of absorption features, the local features at $6.2,11.8$ and $23.1 \mathrm{~km} \mathrm{~s}^{-1}$ and features proposed to be belonging to the Sagittarius spiral arm at 51.1, 62.3 , and $68.8 \mathrm{~km} \mathrm{~s}^{-1}$. We note that for the $\mathrm{H}$ I observations the line widths range between 7.1 and $12.2 \mathrm{~km} \mathrm{~s}^{-1}$ with a spectral resolution of $2.1 \mathrm{~km} \mathrm{~s}^{-1}$, thus leading to an uncertainty in the velocity determination. However, the highest velocity permitted by Galactic rotation is about $60 \mathrm{~km} \mathrm{~s}^{-1}$ in this direction. Therefore the 62.3 and $68.8 \mathrm{~km} \mathrm{~s}^{-1}$ components are most likely associated with a high-velocity (HV) stream. Note also that the CO line emission is brightest at $v_{\mathrm{LSR}}=58 \mathrm{~km} \mathrm{~s}^{-1}$ (Kang et al. 2010), indicating that the ionized and molecular gas have similar velocities and are probably associated with the same radio continuum source.

Using $\mathrm{H}_{2} \mathrm{CO}$ observations, Arnal \& Goss (1985) detected five absorption components at $\sim 52,55,57,66$, and $70 \mathrm{~km} \mathrm{~s}^{-1}$ toward W51e2. As discussed above, the $57 \mathrm{~km} \mathrm{~s}^{-1}$ component seems to be associated with the H II region. The $62 \mathrm{~km} \mathrm{~s}^{-1} \mathrm{com}-$ ponent detected in $\mathrm{H} \mathrm{I}$ and not in $\mathrm{H}_{2} \mathrm{CO}$ is understood to be a $\mathrm{H}$ I cloud associated with W51e2 (G49.5-0.4). $\mathrm{H}_{2} \mathrm{CO}$ absorption between 66 and $70 \mathrm{~km} \mathrm{~s}^{-1}$ and $\mathrm{HI}$ absorption at $68.8 \mathrm{~km} \mathrm{~s}^{-1} \mathrm{im}$ ply that the HV stream is in front of W51e. In the large-scale ${ }^{12} \mathrm{CO}$ and ${ }^{13} \mathrm{CO}$ channel maps, the emission feature in the 66 to $70 \mathrm{~km} \mathrm{~s}^{-1}$ velocity range traces a filamentary structure that extends across a region $\sim 1^{\circ}$ in size that contains most of the $\mathrm{H}$ II regions associated with W51. This is consistent with the triggered star formation scenario that is due to the interaction of the filament with the main cloud, as proposed by Carpenter \& Sanders (1998). Recently, Kang et al. (2010) compared the 

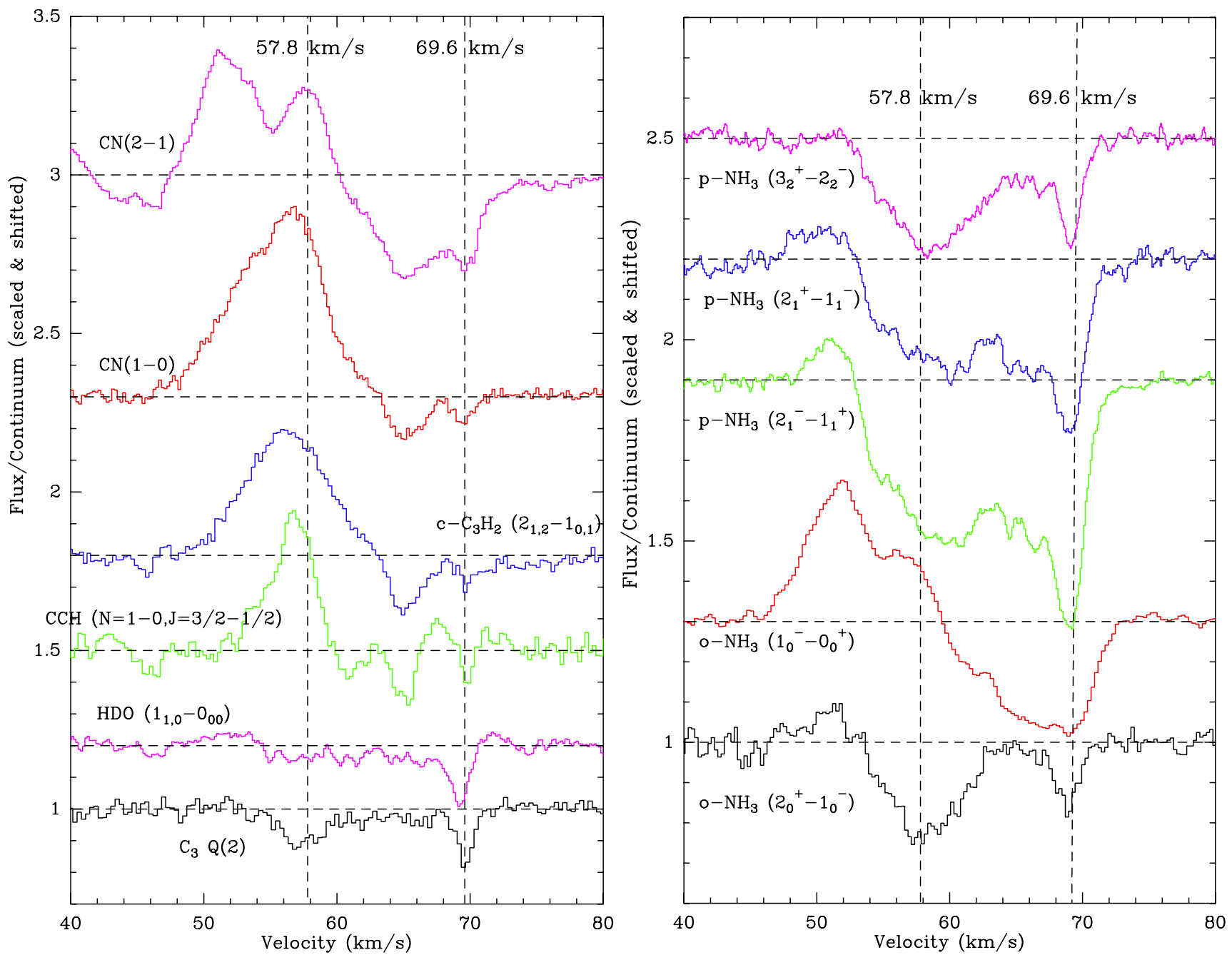

Fig. 3. Left: comparison of velocity structure seen in $\mathrm{C}_{3}$ spectra with that seen in transitions of $\mathrm{CCH}, \mathrm{c}-\mathrm{C}_{3} \mathrm{H}_{2}, \mathrm{HDO}$, and $\mathrm{CN}$. The $\mathrm{H}_{2}^{18} \mathrm{O}$ spectrum along with a Gaussian fit are taken from Flagey et al. (2013). Right: multiple transitions of $\mathrm{NH}_{3}$ observed with Herschel. The dashed vertical lines correspond to the 57.8 and $69.6 \mathrm{~km} \mathrm{~s}^{-1}$ components as found in the $\mathrm{C}_{3}$ observations (see Table 4).

molecular cloud morphology with the distribution of IR and radio continuum sources and found associations between molecular clouds and young stellar objects listed in Spitzer IR catalogs. Star formation in the W51 complex appears to have occurred in a long and large filament along with the $\mathrm{H}$ II regions. Young stellar objects along the filament are younger and more massive than those in the surrounding regions (Carpenter \& Sanders 1998). Cloud-cloud collisions may compress the interface region between two colliding clouds and initiate star formation. The molecular clouds at this interface are heated, but the molecular clouds on the trailing side remain cold. The diffuse emission from the giant molecular cloud truncates at the location of the $68 \mathrm{~km} \mathrm{~s}^{-1}$ cloud for velocities higher than $63 \mathrm{~km} \mathrm{~s}^{-1}$. The W51 cloud and filament are probably at a common distance since such an interface is unlikely to result from the chance superposition of unrelated molecular clouds.

Figure 2 shows the observed $C_{3}$ spectra normalized to the (single-sideband) continuum level. We identify two major absorption dips around 58 and $70 \mathrm{~km} \mathrm{~s}^{-1}$ in the spectra of all three transitions of $\mathrm{C}_{3}$. Figure 3 shows the observed spectra of single or multiple transitions of $\mathrm{CCH}, \mathrm{c}-\mathrm{C}_{3} \mathrm{H}_{2}, \mathrm{NH}_{3}, \mathrm{CN}, \mathrm{HDO}$, and $\mathrm{H}_{2}^{18} \mathrm{O}$ along with the $\mathrm{C}_{3}(Q(2))$ spectrum for comparison. The $\mathrm{H}_{2}^{18} \mathrm{O} 1_{1,1}-0_{0,0}$ spectrum was observed with Herschel/HIFI as part of the PRISMAS program and has been presented by Flagey et al. (2013).

While the $58 \mathrm{~km} \mathrm{~s}^{-1}$ component appears either in emission or in absorption, depending on the transitions, the narrow $70 \mathrm{~km} \mathrm{~s}^{-1}$ feature can be seen only as a deep absorption feature for the different species presented here. The absorption dip at $58 \mathrm{~km} \mathrm{~s}^{-1}$ is broad and most likely is a combination of the $57 \mathrm{~km} \mathrm{~s}^{-1}$ component associated with the $\mathrm{H}$ II region W51e 2 and the $\mathrm{H}$ I cloud at $62 \mathrm{~km} \mathrm{~s}^{-1}$. We note that although the $68 \mathrm{~km} \mathrm{~s}^{-1}$ feature associated with the filament has been relatively easily detected in CO emission (Mufson \& Liszt 1979; Carpenter \& Sanders 1998), the line profiles are much broader than the $70 \mathrm{~km} \mathrm{~s}^{-1} \mathrm{ab}-$ sorption dip detected here. Based on the results of our analyses we show in the following sections that the $70 \mathrm{~km} \mathrm{~s}^{-1}$ absorption feature can only be explained in terms of a high-density $\left(>10^{5} \mathrm{~cm}^{-3}\right)$ clump that formed within the filament.

\section{Estimations of the column and volume densities}

Tables 3 and 4 summarize the basic observational results for $\mathrm{CCH}, \mathrm{c}-\mathrm{C}_{3} \mathrm{H}_{2}, \mathrm{NH}_{3}, \mathrm{HDO}$, and $\mathrm{C}_{3}$. The central velocities, linewidths, and main-beam temperatures of all species are derived by fitting Gaussian components to the $70 \mathrm{~km} \mathrm{~s}^{-1}$ feature of 
Table 3. Basic observational results for $\mathrm{NH}_{3}, \mathrm{HDO}, \mathrm{CCH}$, and $\mathrm{c}-\mathrm{C}_{3} \mathrm{H}_{2}$.

\begin{tabular}{lccccc}
\hline \hline Species & Transition & $\begin{array}{c}T_{\mathrm{c}} \\
\mathrm{K}\end{array}$ & $\begin{array}{c}v_{\mathrm{LSR}} \\
\mathrm{km} \mathrm{s}^{-1}\end{array}$ & $\begin{array}{c}\Delta V \\
\mathrm{~km} \mathrm{~s}^{-1}\end{array}$ & $\begin{array}{c}\int T_{\mathrm{mb}} \mathrm{d} V \\
\mathrm{~K} \mathrm{~km} \mathrm{~s}^{-1}\end{array}$ \\
\hline ortho- $\mathrm{NH}_{3}$ & $1_{0}^{-}-0_{0}^{+}$ & $1.40 \pm 0.04$ & $\ldots$ & $\ldots$ & $\ldots$ \\
& $2_{0}^{+}-1_{0}^{-}$ & $7.40 \pm 0.29$ & $68.83 \pm 0.06$ & $2.03 \pm 0.16$ & $3.44 \pm 0.49$ \\
para- $\mathrm{NH}_{3}$ & $2_{1}^{-}-1_{1}^{+}$ & $7.30 \pm 0.11$ & $68.79 \pm 0.02$ & $3.14 \pm 0.07$ & $15.13 \pm 0.49$ \\
& $2_{1}^{+}-1_{1}^{-}$ & $7.40 \pm 0.29$ & $68.78 \pm 0.04$ & $3.30 \pm 0.10$ & $15.62 \pm 0.92$ \\
& $3_{2}^{+}-2_{2}^{-}$ & $9.80 \pm 0.21$ & $69.07 \pm 0.02$ & $2.08 \pm 0.05$ & $6.23 \pm 0.24$ \\
$\mathrm{HDO}$ & $1_{1,1}-0_{0,0}$ & $7.10 \pm 0.10$ & $69.17 \pm 0.02$ & $1.52 \pm 0.06$ & $1.64 \pm 0.10$ \\
$\mathrm{CCH}$ & $1_{3 / 2,1}-0_{1 / 2,1}$ & $1.70 \pm 0.09$ & $56.69 \pm 0.06$ & $3.50 \pm 0.50$ & $5.40 \pm 0.92^{\star}$ \\
& & $1.70 \pm 0.09$ & $60.76 \pm 0.14$ & $1.30 \pm 0.20$ & $0.53 \pm 0.18$ \\
& & $1.70 \pm 0.09$ & $65.01 \pm 0.09$ & $1.50 \pm 0.20$ & $0.99 \pm 0.23$ \\
$\mathrm{c}-\mathrm{C}_{3} \mathrm{H}_{2}$ & & $1.70 \pm 0.09$ & $69.79 \pm 0.09$ & $0.68 \pm 0.21$ & $0.30 \pm 0.17$ \\
& $2_{1,2}-1_{0,1}$ & $1.00 \pm 0.04$ & $56.17 \pm 0.15$ & $6.75 \pm 0.36$ & $4.77 \pm 0.09^{\star}$ \\
& & $1.00 \pm 0.04$ & $65.26 \pm 0.05$ & $2.63 \pm 0.12$ & $0.78 \pm 0.09$ \\
& & $1.00 \pm 0.04$ & $69.70 \pm 0.15$ & $0.54 \pm 0.20$ & $0.13 \pm 0.09$ \\
\hline
\end{tabular}

Notes. ${ }^{\star}{ }^{\star}$ Lines are detected in emission.

Table 4. Parameters derived for $\mathrm{C}_{3}$ by simultaneous Gaussian fitting of all observed transitions ${ }^{a}$.

\begin{tabular}{lccrr}
\hline \hline Transition & $\begin{array}{c}T_{\mathrm{c}}{ }^{b} \\
{[\mathrm{~K}]}\end{array}$ & $\begin{array}{c}v_{\mathrm{LSR}} \\
{\left[\mathrm{km} \mathrm{s}^{-1}\right]}\end{array}$ & $\begin{array}{r}\int \tau \mathrm{d} v \\
{\left[\mathrm{~km} \mathrm{~s}^{-1}\right]}\end{array}$ & $\begin{array}{r}N_{\mathrm{l}} \\
{\left[10^{13} \mathrm{~cm}^{-2}\right]}\end{array}$ \\
\hline$P(4)$ & $10.5 \pm 1.3$ & 57.8 & $0.14 \pm 0.05$ & $3.5 \pm 1.0$ \\
& & 69.6 & $0.11 \pm 0.02$ & $2.7 \pm 0.5$ \\
$Q(2)$ & $10.5 \pm 0.6$ & 57.8 & $0.30 \pm 0.05$ & $2.5 \pm 0.3$ \\
& & 69.6 & $0.31 \pm 0.03$ & $2.6 \pm 0.2$ \\
$Q(4)$ & $10.7 \pm 0.7$ & 57.8 & $0.49 \pm 0.07$ & $4.1 \pm 0.4$ \\
& & 69.6 & $0.23 \pm 0.02$ & $1.9 \pm 0.2$ \\
\hline
\end{tabular}

Notes. ${ }^{(a)}$ The fitted velocity components are component 1: $v_{\mathrm{LSR}}=$ $57.81 \pm 0.01 \mathrm{~km} \mathrm{~s}^{-1}, \Delta V=4.72 \pm 0.38 \mathrm{~km} \mathrm{~s}^{-1}$, component $2: v_{\mathrm{LSR}}=$ $69.55 \pm 0.04 \mathrm{~km} \mathrm{~s}^{-1}, \Delta V=1.41 \pm 0.10 \mathrm{~km} \mathrm{~s}^{-1}$. ${ }^{(b)}$ Single sideband continuum main beam temperatures $\left(\eta_{\mathrm{mb}}=0.69\right)$.

the observed spectra. In the following analyses, we assumed that the foreground cloud at $70 \mathrm{~km} \mathrm{~s}^{-1}$ completely fills the beam.

\section{1. $C_{3}$ LTE modeling}

We fitted the three detected transitions of $\mathrm{C}_{3}$ simultaneously using two Gaussian components corresponding to two velocity components per transition. The fit derives a common $v_{\text {LSR }}$ and full width at half maximum $(\Delta v)$ for each of the velocity components for all transitions as well as the strength of the absorption dips. Table 4 presents the single-sideband continuum levels (estimated from double-sideband temperatures assuming a sideband gain ratio of unity) and the opacities derived from the Gaussian fitting.

Assuming LTE we used the level-specific column densities derived from the $\mathrm{C}_{3}$ absorption dips (Table 4) following the rotation diagram method. For the $58 \mathrm{~km} \mathrm{~s}^{-1}$ component we estimate the rotation temperatures $\left(T_{\text {rot }}\right)$ and total column density $\left(N\left(\mathrm{C}_{3}\right)\right)$ to be $10 \mathrm{~K}$ and $1.0 \times 10^{14} \mathrm{~cm}^{-2}$, respectively, for the $70 \mathrm{~km} \mathrm{~s}^{-1}$ component these are $10 \mathrm{~K}$ and $6.0 \times 10^{13} \mathrm{~cm}^{-2}$, respectively. Since the determination of $T_{\text {rot }}$ and the column density involves fitting of only two datapoints with associated uncertainties to estimate two parameters, the derived parameters are expected to have very large uncertainties. Additionally, since the far-infrared continuum radiation is known to contribute significantly to the
Table 5. Derived parameters for the absorption components detected in $\mathrm{CCH}$ and $\mathrm{c}-\mathrm{C}_{3} \mathrm{H}_{2}$ spectra.

\begin{tabular}{lcccc}
\hline \hline Species & $\begin{array}{c}v_{\mathrm{LSR}} \\
\mathrm{km} \mathrm{s}^{-1}\end{array}$ & $\begin{array}{c}\Delta v \\
\mathrm{~km} \mathrm{~s}^{-1}\end{array}$ & $\tau$ & $\begin{array}{c}N \\
\mathrm{~cm}^{-2}\end{array}$ \\
\hline $\mathrm{CCH}$ & 69.8 & 0.7 & 0.37 & $4.9 \times 10^{13}$ \\
& 65.0 & 1.5 & 0.52 & $1.3 \times 10^{14}$ \\
& 60.8 & 1.3 & 0.28 & $6.0 \times 10^{13}$ \\
$\mathrm{c}^{-} \mathrm{C}_{3} \mathrm{H}_{2}$ & 69.7 & 0.54 & 0.23 & $7.5 \times 10^{11}$ \\
& 65.3 & 2.63 & 0.35 & $5.5 \times 10^{12}$ \\
\hline
\end{tabular}

excitation of $\mathrm{C}_{3}$ (Roueff et al. 2002), an accurate estimate of temperature can only be obtained using rigorous radiative transfer treatment. We therefore only used the derived total column densities from the LTE analysis.

\subsection{Column density estimates from LTE modeling: $\mathrm{CCH}$ and $\mathrm{c}-\mathrm{C}_{3} \mathrm{H}_{2}$}

We used the IRAM-30 m c- $\mathrm{C}_{3} \mathrm{H}_{2}$ observations presented by Gerin et al. (2011) and the IRAM-PdBI CCH observations to compute the column densities in the absorption components along the line of sight to W51e2 using the CASSIS software ${ }^{3}$.

For $\mathrm{CCH}$ we used the average (taken over $5^{\prime \prime}$ ) spectrum centered on the HIFI position observed with PdBI for modeling. We detected the $70 \mathrm{~km} \mathrm{~s}^{-1}$ absorption feature in only one transition of each of $\mathrm{CCH}$ and $\mathrm{c}-\mathrm{C}_{3} \mathrm{H}_{2}$. Since both $\mathrm{CCH}$ and $\mathrm{c}-\mathrm{C}_{3} \mathrm{H}_{2}$ lines at $70 \mathrm{~km} \mathrm{~s}^{-1}$ appear in emission instead of absorption for $T_{\text {ex }}>3 \mathrm{~K}$, we derived the molecular column densities for each velocity component in absorption assuming that the excitation temperature equals the $\mathrm{CMB}$. The ratio of the column densities of $\mathrm{CCH}$ and $\mathrm{c}-\mathrm{C}_{3} \mathrm{H}_{2}$ in the $70 \mathrm{~km} \mathrm{~s}^{-1}$ component $(\sim 67)$ is similar to the value found in the extended ridge component $\left(N\left(\mathrm{H}_{2}\right)=\right.$ $3 \times 10^{23} \mathrm{~cm}^{-2}$ ) of the OMC-1 region (Blake et al. 1987, see Sect. 7.1).

\subsection{Volume density estimates from $\mathrm{NH}_{3}$}

Because the ortho- $\mathrm{NH}_{3}$ fundamental transition at $572.5 \mathrm{GHz}$ are highly optically thick, with blending in the line of sight (see

\footnotetext{
3 A software developed by IRAP-UPS-CNRS: http://cassis. irap.omp.eu
} 
Table 6. Non-LTE (RADEX) modeling of $\mathrm{NH}_{3}$ and $\mathrm{CN}$.

\begin{tabular}{lccrcr}
\hline \hline Species & $\begin{array}{c}v_{\mathrm{LSR}} \\
\mathrm{km} \mathrm{s}^{-1}\end{array}$ & $\begin{array}{c}\Delta v \\
\mathrm{~km} \mathrm{~s}^{-1}\end{array}$ & $\begin{array}{r}\mathrm{T}_{\mathrm{kin}} \\
\mathrm{K}\end{array}$ & $\begin{array}{c}N \\
\mathrm{~cm}^{-2}\end{array}$ & $\begin{array}{r}n\left(\mathrm{H}_{2}\right) \\
\mathrm{cm}^{-3}\end{array}$ \\
\hline $\mathrm{CN}$ & 69.5 & 0.6 & 6.0 & $5.5 \times 10^{13}$ & $10^{5}$ \\
$\mathrm{NH}_{3}$ & 68.8 & 2.0 & 30.0 & $8.4 \times 10^{13}$ & $5 \times 10^{5}$ \\
\hline
\end{tabular}

Notes. The $\mathrm{H}_{2}$ density and kinetic temperature found are valid to within $50 \%$.

Fig. 3), the $1168.5,1214.8,1215.2$, and $1763.8 \mathrm{GHz}$ transitions were used to separate the different components and extract the $69.2 \pm 0.2 \mathrm{~km} \mathrm{~s}^{-1}$ component. The results from the Gaussian fitting procedure are presented in Table 3. Indeed, the $572 \mathrm{GHz}$ transition is affected by blending and saturation effects and does not allow us to obtain a reliable estimate for the ortho-to-para ratio in the $70 \mathrm{~km} \mathrm{~s}^{-1}$ cloud, in addition to $n\left(\mathrm{H}_{2}\right)$ and $T_{\text {kin }}$. Furthermore, we do not have additional observations that might be used to identify the $\mathrm{NH}_{3}$ formation process (temperature and mechanism in particular) (Persson et al. 2012) and the $\mathrm{H}_{2}$ ortho-para ratio (Faure et al. 2013), both of which are known to result in an $\mathrm{NH}_{3}$ ortho-para ratio significantly different from unity. We assumed the ortho-to-para ratio to be equal to the statistical equilibrium value of unity. The ortho- and para- $\mathrm{NH}_{3}$ collisional coefficients with para- $\mathrm{H}_{2}$ (Danby et al. 1988) were used in the non-LTE RADEX computations within CASSIS. In the CASSIS database the ortho and para separation is provided with partition functions for temperatures between 3 and $300 \mathrm{~K}$. Note that there is a discrepancy between the fitted line widths of the two $2{ }_{1}-1_{1}\left(\sim 3 \mathrm{~km} \mathrm{~s}^{-1}\right)$ transitions and the $2_{0}^{+}-1_{0}^{-}$and $3_{2}^{+}-2_{2}^{-}$ $\left(2.0 \mathrm{~km} \mathrm{~s}^{-1}\right)$ transitions. However, we argue that the line widths of the $2{ }_{1}-1_{1}$ transitions are more affected by additional components in its wings than the more isolated velocity components of the $3_{2}^{+}-2_{2}^{-}$and $2_{0}^{+}-1_{0}^{-}$transitions. We therefore varied the line width around a value of $2 \mathrm{~km} \mathrm{~s}^{-1}$ in the modeling. The bestfitting model for the $70 \mathrm{~km} \mathrm{~s}^{-1}$ cloud is obtained for a line width of $2 \mathrm{~km} \mathrm{~s}^{-1}$ with an $\mathrm{H}_{2}$ density of $5 \times 10^{5} \mathrm{~cm}^{-3}$, and a kinetic temperature of $\sim 30 \mathrm{~K}$, with a column density of $4.2 \times 10^{13} \mathrm{~cm}^{-2}$ for each symmetry. Hence the total estimated column density of $\mathrm{NH}_{3}$ is $8.4 \times 10^{13} \mathrm{~cm}^{-2}$.

\subsection{Volume density estimates from $\mathrm{CN}$}

We observed the $J=1-0$ and 2-1 transitions of $\mathrm{CN}$ using the IRAM $30 \mathrm{~m}$. CN 1-0 is a four component multiplet that appears in absorption against the $0.95 \mathrm{~K}$ continuum, and $\mathrm{CN} 2-1$ is a six component multiplet, four components of which appear in absorption against the $2.1 \mathrm{~K}$ continuum (the other components are blended with the emission components at the source velocity). Figure 4 shows the observed spectra along with the bestfit LTE model for $T_{\mathrm{ex}}=3 \mathrm{~K}, v_{\mathrm{LSR}}=69.5 \mathrm{~km} \mathrm{~s}^{-1}$, line width of $0.6 \mathrm{~km} \mathrm{~s}^{-1}$ and a total column density of $5.8 \times 10^{13} \mathrm{~cm}^{-2}$. Recently, the collision rates have been computed with $\mathrm{p}-\mathrm{H}_{2}$ taking into account all the $\mathrm{CN}$ hyperfine levels (Kalugina et al. 2012). Assuming the line width of the $70 \mathrm{~km} \mathrm{~s}^{-1}$ component to be $0.6 \mathrm{~km} \mathrm{~s}^{-1}$, we ran a non-LTE model (RADEX) to reproduce the seven $\mathrm{CN}$ hyperfine components seen in absorption and found a density of $n\left(\mathrm{H}_{2}\right) \sim 10^{5} \mathrm{~cm}^{-3}$ (assuming that $\mathrm{H}_{2}$ is mainly in its para form) and a kinetic temperature of $\sim 6 \mathrm{~K}$ with a column density of $\sim 5.5 \times 10^{13} \mathrm{~cm}^{-2}$. Both the density and temperature required to explain the observed $\mathrm{CN}$ spectra are lower than the values required to explain the observed ammonia transitions (see Table 6). A possible reason for the low temperature
Table 7. Abundances of all chemical species estimated relative to the observed CCH column density in the $70 \mathrm{~km} \mathrm{~s}^{-1}$ clump.

\begin{tabular}{|c|c|c|}
\hline \multirow[t]{2}{*}{ Species } & \multicolumn{2}{|c|}{$70 \mathrm{~km} \mathrm{~s}^{-1}$ clump } \\
\hline & $\begin{array}{l}N \\
10^{13} \mathrm{~cm}^{-2}\end{array}$ & $X^{a}$ \\
\hline $\mathrm{C}_{3}$ & 6.0 & 1.2 \\
\hline $\mathrm{CCH}$ & 4.9 & 1.0 \\
\hline$c-\mathrm{C}_{3} \mathrm{H}_{2}$ & 0.08 & 0.016 \\
\hline $\mathrm{NH}_{3}$ & 8.4 & 1.72 \\
\hline $\mathrm{CN}$ & 5.5 & 1.12 \\
\hline HDO & 0.15 & 0.031 \\
\hline
\end{tabular}

Notes. (a) Abundance relative to $N(\mathrm{CCH})=4.9 \times 10^{13} \mathrm{~cm}^{-2}$. ${ }^{(b)}$ Mookerjea et al. (2012).

derived can be the dilution of the continuum within the beam, combined with a cold foreground component that is covering part of the continuum. Assuming a higher continuum value (e.g. $1.7 \mathrm{~K}$, as found for $\mathrm{CCH}$ ) would lead to a $10 \mathrm{~K}$ kinetic temperature for the $70 \mathrm{~km} \mathrm{~s}^{-1}$ component. We therefore consider $6 \mathrm{~K}$ to be a lower limit for the temperature derived from $\mathrm{CN}$. However, given the significantly different temperatures $(30 \mathrm{~K})$ and densities $\left(5 \times 10^{5} \mathrm{~cm}^{-3}\right)$ found for $\mathrm{NH}_{3}$ along with the extremely narrow line width of the $\mathrm{CN}$ lines compared with those found in $\mathrm{NH}_{3}, \mathrm{C}_{3}$, and $\mathrm{HDO}$, it is very likely that $\mathrm{CN}$ arises from a colder and less dense envelope, possibly giving rise to $\mathrm{CCH}$ and c- $\mathrm{C}_{3} \mathrm{H}_{2}$ as well.

\section{5. $H D O$}

Most of the compact $\mathrm{H}$ II regions observed within the PRISMAS key program present a broad absorption profile in the $893 \mathrm{GHz}$ fundamental transition of HDO at the source $v_{\text {LSR }}$. The W51 region is the only high-mass source where this transition appears as a deep narrow $\left(<2 \mathrm{~km} \mathrm{~s}^{-1}\right)$ feature at a velocity higher than the source velocity. The other HDO fundamental transition at $464 \mathrm{GHz}$ has also been observed but the high-velocity absorption feature is not detected reliably above the noise of the spectrum $(\mathrm{rms}=0.13 \mathrm{~K})$. From the narrow component observed at $893 \mathrm{GHz}$, we can state that this component is clearly localized and probably not extended along the line of sight. Both HDO ground-state transitions have high critical densities $\left(>10^{7} \mathrm{~cm}^{-3}\right)$ and do not provide strong constraints on the kinetic temperature or density for the range found with the $\mathrm{NH}_{3}$ and $\mathrm{CN}$ analysis. Because the $\mathrm{NH}_{3}$ transitions trace a dense region in the filament, which is also more likely to emit in HDO than the lower density and lower temperature region that is traced by $\mathrm{CN}$, we estimate for $T_{\text {kin }}=30 \mathrm{~K}$ and $n\left(\mathrm{H}_{2}\right)=5 \times 10^{5} \mathrm{~cm}^{-3}$ the total HDO column density under non-LTE to be $1.5 \times 10^{12} \mathrm{~cm}^{-2}$, which is similar to the LTE value with an excitation temperature of $3 \mathrm{~K}$.

\section{Chemical modeling of abundance}

In the absence of a direct estimate for the $\mathrm{H}_{2}$ column density for the $70 \mathrm{~km} \mathrm{~s}^{-1}$ clump, we expressed the abundances of all the observed molecules $\mathrm{C}_{3}, \mathrm{c}-\mathrm{C}_{3} \mathrm{H}_{2}, \mathrm{NH}_{3}, \mathrm{CN}$, and HDO (Table 7) relative to the column density of $\mathrm{CCH}$ and compared this with the results of the Ohio State University (OSU) gas-grain chemical model with a warm-up (Hasegawa et al. 1992; Garrod \& Herbst 2006). A previous version of this model has already been used to interpret the abundance of $\mathrm{C}_{3}$ in the warm envelopes of hot cores 

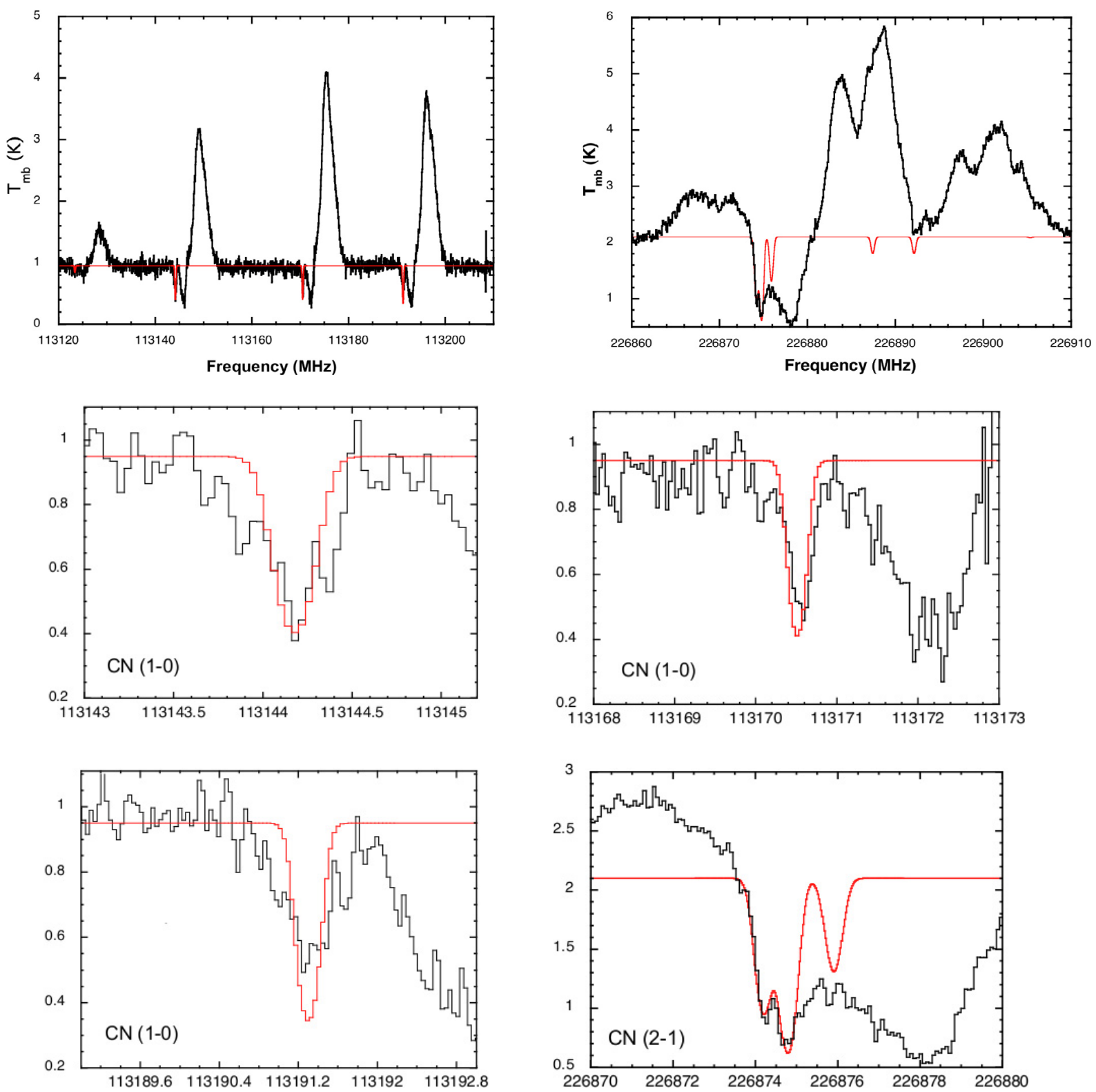

Fig. 4. Top panels: observed spectra of CN 1-0 (left) and CN 2-1 (right) shown in black. We also show the best-fit LTE model (using the CASSIS software) corresponding to $T_{\mathrm{ex}}=3 \mathrm{~K}, v_{\mathrm{LSR}}=69.5 \mathrm{~km} \mathrm{~s}^{-1}, \Delta v=0.6 \mathrm{~km} \mathrm{~s}^{-1}$, and $N_{\mathrm{CN}}=5.8 \times 10^{13} \mathrm{~cm}^{-2}$. The data and the relevant best-fit model spectra are enlarged in the four panels below. The details of the $\mathrm{CN}(1-0)$ transitions are middle row left: $1,1 / 2,1 / 2-0,1 / 2,3 / 2$ and right: 1, 1/2, $3 / 2-0,1 / 2,1 / 2$; bottom row left: $1,1 / 2,3 / 2-0,1 / 2,3 / 2$.

such as W31C, W49N, and DR21OH (Mookerjea et al. 2010, 2012). While the $70 \mathrm{~km} \mathrm{~s}^{-1}$ component is not directly associated to the hot core, the necessity of grain warm-up in explaining the abundance of $\mathrm{C}_{3}$ in dense environments was illustrated by Hassel et al. (2011). As explained by Mookerjea et al. (2012), formation of $\mathrm{C}_{3}$ in dense environments cannot proceed via the gas-phase pathway as in diffuse clouds and requires desorption (either by radiation or by cosmic ray) of $\mathrm{CH}_{4}$ from icy grain mantles to produce $\mathrm{C}_{2} \mathrm{H}_{2}$, which reacts further to produce $\mathrm{C}_{3}$.

The chemical model is adapted from models of the hot-core phase of protostellar sources (Viti et al. 2004; Garrod \& Herbst 2006). Standard hot-core chemistry requires that dust grains build up icy mantles at early times, when the temperature is low $(\sim 10 \mathrm{~K})$ and the core is in a state of collapse (Brown et al. 1988). Later, the formation of a nearby protostar quickly warms the gas and dust (warm-up phase), re-injecting the grain mantle material into the gas phase, and stimulating a rich chemistry. Thus, hot-core models can involve three distinct phases: (i) the collapse of a cold clump of interstellar gas to high densities; (ii) heating of this clump, and the subsequent evaporation of grain mantles; and (iii) the hot-core phase. The present model examines (ii), the warm-up phase, and starts from the time at which the clump has collapsed to the high density and considers heating of the constant density clump and subsequent grain surface and gas-phase chemistry, corresponding to Phase 2 of Viti et al. (2004), based on the approximation of Hassel et al. (2008). In the warm-up approach, a one-point parcel of material undergoes an initially cold period of $T_{0}=10 \mathrm{~K}$ with a duration of $t=10^{5} \mathrm{yr}$, followed by a gradual temperature increase to a maximum temperature, $T_{\max }$. A heating time-scale of $t_{\mathrm{h}}=0.2 \mathrm{Myr}$ was adopted following Garrod \& Herbst (2006), in which the temperature reaches $T_{\max }$ by 0.3 Myr. In the warm-up, the dust and gas temperatures are assumed to be equal and follow the same evolution. Unlike the hot-core models, it is unclear whether the filament harbors star 


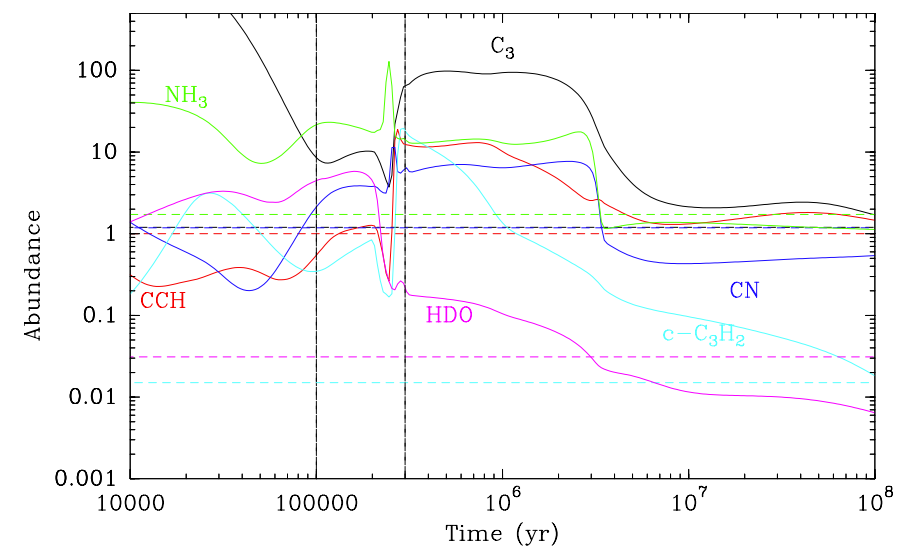

Fig. 5. Calculated abundances of different species relative to the observed column density of $\mathrm{CCH}$, as a function of time for a constant density $n\left(\mathrm{H}_{2}\right)=5 \times 10^{5} \mathrm{~cm}^{-3}$ and a warm-up to $T_{\max }=30 \mathrm{~K}$ from $10 \mathrm{~K}$, where the warm-up and other conditions are described in Sect. 6. The dashed horizontal lines correspond to the observed abundances and are color-coded with the calculated abundances. The vertical lines at $t=0.1$ and $0.3 \mathrm{Myr}$ indicate the onset and end of warm-up of grains, respectively.

formation. We therefore considered values of $T_{\max }$ that represent relatively mild warming. The physical parameters adopted are the same as used by Garrod et al. (2007) and Hassel et al. (Table 1 ; 2008), including a canonical value for cosmic-ray ionization rate $\zeta=1.3 \times 10^{-17} \mathrm{~s}^{-1}$. The simplified warm-up and the estimates for the parameters were previously adopted by Hassel et al. (2008) following Garrod \& Herbst (2006) to investigate the details of chemical evolution without a strict dependence on a particular dynamical model, and have since been used in Hassel et al. (2011) and Mookerjea et al. (2012). Although the dense clump may not contain a hot core, we adopted the same approach in this model for the same general reason, and in particular to incorporate reactions involving D-bearing species into a familiar modeling procedure.

The gas-grain chemistry reaction network has been expanded since the previous $\mathrm{C}_{3}$ models to include HDO, and now comprises 7882 reactions involving 735 gaseous and surface species. We included the major gaseous D-exchange reactions of the UMIST database (McElroy et al. 2013), the major surface reactions identified by Stantcheva \& Herbst (2003), and gas-phase reactions related to HDO using the approach of Aikawa et al. (2012). The initial chemical abundances were adopted from Garrod et al. (2007), and include the initial abundance [HD] = $1.5 \times 10^{-5} \times\left[\mathrm{H}_{2}\right]$ (Osamura et al. 2005).

The observed multiple transitions of $\mathrm{NH}_{3}$ provide the only independent estimate of the density and temperature of the filament that gives rise to the $70 \mathrm{~km} \mathrm{~s}^{-1}$ component. Here we present a comparison of the observed abundances for all molecules considered with the abundances predicted by the chemical model for which $T_{\max }$ is $30 \mathrm{~K}$ and the molecular hydrogen density is $5 \times$ $10^{5} \mathrm{~cm}^{-3}$ (Fig. 5). The predicted abundances of all molecules are significantly different from the observed values at times earlier than 3 Myr. The abundances of $\mathrm{NH}_{3}, \mathrm{CCH}$, and $\mathrm{CN}$ predicted by the model match the observed abundances well at all times beyond $t=3.5 \mathrm{Myr}$. The model abundance of $\mathrm{c}-\mathrm{C}_{3} \mathrm{H}_{2}$, is significantly higher than the observed abundance, at all times. Previous models using a similar chemical network have also demonstrated a larger calculated abundance than the observation (Fig. 4; Hassel et al. 2011). It is also possible that $\mathrm{c}-\mathrm{C}_{3} \mathrm{H}_{2}$ arises in a colder and less dense envelope ( similar to $\mathrm{CN}$ ), than the values of $n\left(\mathrm{H}_{2}\right)$ and $T_{\max }$ used in the model presented here. Beyond $5 \mathrm{Myr}$ the model abundance of $\mathrm{C}_{3}$ approaches the observed abundance for all times up to $t=70 \mathrm{Myr}$. For $\mathrm{C}_{3}$ the model abundance is within a factor of 3 of the observed abundance for all times later than $5 \mathrm{Myr}$. The HDO model abundance is consistent to within a factor of 2.5 of the observed abundance between 3.5 to $30 \mathrm{Myr}$ and diverges farther at later times.

\section{Discussion}

In the direction of W51e2 we have for the first time detected $\mathrm{HDO}$ and $\mathrm{C}_{3}$ in a dense molecular clump at $70 \mathrm{~km} \mathrm{~s}^{-1}$ that is not directly associated with hot star-forming cores.

$\mathrm{C}_{3}$ in dense clouds has so far only been detected in the warm envelopes associated with star-forming cores. Abundance of $\mathrm{C}_{3}$ in dense clouds is explained via warming-up of grain mantles to release $\mathrm{CH}_{4}$ and subsequently $\mathrm{C}_{3} \mathrm{H}_{2}$, the first step in the chemical pathway for the formation of $\mathrm{C}_{3}$, as explained in detail in Hassel et al. (2011) and Mookerjea et al. (2012). Thus, detection of $\mathrm{C}_{3}$ in the dense clump at $70 \mathrm{~km} \mathrm{~s}^{-1}$ otherwise not identified as a star-forming region is as surprising as the detection of deuterated water in the cloud that is physically not related to the W51 region. HDO is most likely formed in the ice mantles within cold $(<20 \mathrm{~K})$ translucent clouds (Cazaux et al. 2011) in regions shielded from UV radiation. Indeed, at $A_{v} \gtrsim 3$ (Whittet et al. 1988), species from the gas phase accrete onto dust grains and initiate ice formation. After the formation of water and deuterated water in the ice mantles in the star formation scenario, the environment undergoes gravitational collapse and the local density increases, leading to the formation of a protostar. The surrounding medium then heats up and releases the icy mantles into the gas phase, leading to a significant HDO abundance in the gas phase (Coutens et al. 2012, 2013b). Based on the nonLTE modeling of the five observed $\mathrm{NH}_{3}$ transitions, we characterize the region of the filament that gives rise to the $70 \mathrm{~km} \mathrm{~s}^{-1}$ feature as having $T_{\text {kin }}=30 \mathrm{~K}$ and $n\left(\mathrm{H}_{2}\right)=5 \times 10^{5} \mathrm{~cm}^{-3}$. The abundances predicted by the grain warm-up chemical model reproduce the observed abundances to within factors of 2 to 3 at all times later than 3.5 Myr for all species except for $\mathrm{c}-\mathrm{C}_{3} \mathrm{H}_{2}$.

\section{1. $\mathrm{CCH} / \mathrm{c}-\mathrm{C}_{3} \mathrm{H}_{2}$ ratio in the clump}

Previous observations of $\mathrm{CCH}$ and $\mathrm{c}-\mathrm{C}_{3} \mathrm{H}_{2}$ toward dense dark clouds and Photon Dominated Regions (PDRs) derived a tight correlation between the two species. The ratio $N(\mathrm{CCH}) /$ $N\left(\mathrm{c}-\mathrm{C}_{3} \mathrm{H}_{2}\right)$ is $\sim 61$ in OMC-1 (Blake et al. 1987) and it ranges between 10 and 25 in PDRs such as Horsehead nebula and IC63 whereas in dark clouds such as TMC-1, and L134N the ratio is $\sim 1$ (Teyssier et al. 2004). For the dense clump detected toward W51e2 the observed $N(\mathrm{CCH}) / N\left(\mathrm{c}-\mathrm{C}_{3} \mathrm{H}_{2}\right)$ of 67 is closer to the value observed in the dense active star-forming region OMC-1 than to the values seen in UV-illuminated clouds and dark clouds.

\subsection{Deuterium enrichment in the clump}

Using Herschel/HIFI, a number of transitions of $\mathrm{H}_{2} \mathrm{O}$ and $\mathrm{H}_{2}{ }^{18} \mathrm{O}$ species corresponding to a range of excitations have been observed for the first time. The $70 \mathrm{~km} \mathrm{~s}^{-1}$ feature is detected unambiguously in the $\mathrm{H}_{2} \mathrm{O} 2_{0,2}-1_{1,1}$ transition as well as the $\mathrm{H}_{2}{ }^{18} \mathrm{O} 1_{1,1}-0_{0,0}$ transition (Flagey et al. 2013). However, an accurate determination of the total $\mathrm{H}_{2} \mathrm{O}$ column density is compromised by the crowded environment along the line of sight with 
high optical depth, as well as by partial blending of the components. The column density for the less abundant para- $\mathrm{H}_{2}{ }^{18} \mathrm{O}$ species is estimated to be $(7 \pm 1) 10^{11} \mathrm{~cm}^{-2}$ for the $70 \mathrm{~km} \mathrm{~s}^{-1}$. The abundance ratio between ${ }^{16} \mathrm{O}$ and ${ }^{18} \mathrm{O}$ is commonly accepted to be $\sim 560$ (Wilson \& Rood 1994), although significant variation has been observed throughout the Galaxy. Accordingly, we estimate a $\mathrm{D} / \mathrm{H}$ ratio in water vapor of about $9.6 \times 10^{-4}$ (assuming an ortho-para ratio of 3), similar to the value found in Orion KL by Neill et al. (2013). This ratio has also been estimated from HDO observations of the low-mass protostar IRAS 16293-2422 (Coutens et al. 2012, 2013a). The derived values of $\mathrm{HDO} / \mathrm{H}_{2} \mathrm{O}$ were $1.8 \times 10^{-2}$ in the hot core, $5 \times 10^{-3}$ in the cold envelope, and about $4.8 \times 10^{-2}$ in the external photodesorption layer. The value estimated from our analysis is consistent with the value found in the cold envelope of the IRAS 16293-2422 low-mass star-forming region, in which the ices are not affected by thermal desorption or photo-desorption by the FUV field. For the best-fit grain warm-up model that reproduces the observed abundances of the other molecules, at times later than 3.5 Myr the $\mathrm{HDO} / \mathrm{H}_{2} \mathrm{O}$ ratio drops from 0.002 to 0.001 for an initial $\mathrm{HD} / \mathrm{H}_{2}$ ratio of $1.5 \times 10^{-5}$. The calculated $\mathrm{HDO} / \mathrm{H}_{2} \mathrm{O}$ ratio is consistent with the observed ratio of $9.6 \times 10^{-4}$. Although HDO forms in the medium density, pre-collapse phase, it mostly remains on grain mantles, since the nonthermal desorption mechanisms have a limited efficiency. In these models, which reach only $30 \mathrm{~K}$, the release of HDO into the gas phase is attributed to nonthermal processes related to cosmic-ray interactions rather than warming up or direct FUV photodesorption. Although the chemical model predicts an $\mathrm{HDO} / \mathrm{H}_{2} \mathrm{O}$ ratio consistent with the observed value for $t>3.5 \mathrm{Myr}$, it overestimates the $\mathrm{HDO} / \mathrm{H}_{2} \mathrm{O}$ ratio by more than a factor of 10 for earlier times $t<1 \mathrm{Myr}$. The calculated $\mathrm{HDO} / \mathrm{H}_{2} \mathrm{O}$ ratio is substantially enriched above the initial $\mathrm{HD} / \mathrm{H}_{2}$ ratio, primarily because of the grain surface formation route of HDO. The ratio is furthermore enriched above the observed value because of the lower column density of $\mathrm{H}_{2} \mathrm{O}$ calculated by the chemical models. We explored the possibility of enhanced cosmic-ray ionization rates. For enhanced cosmic ray rates the chemical models produce abundances very different from the observed values for all molecules. This result for the $70 \mathrm{~km} \mathrm{~s}^{-1}$ cloud is in contrast to the higher values of $\zeta$ deduced by Indriolo et al. (2012) along the same line of sight for the absorption features arising because of diffuse foreground. This implies that the absorbing gas is not diffuse, but is dense with a standard value of $\zeta$.

\subsection{Possible origin of the clump}

The $70 \mathrm{~km} \mathrm{~s}^{-1}$ absorption dip caused by the foreground material in the direction of W51e2, although not associated with a star-forming core, shows chemical abundances typically found in the envelopes of low-mass protostellar candidates. We propose that the feature arises in a dense $\left(n\left(\mathrm{H}_{2}\right)=(1-5) \times 10^{5} \mathrm{~cm}^{-3}\right)$ and cold $(10-30 \mathrm{~K})$ clump that is formed within the much larger scale filament (detected in $\mathrm{CO}$ ) deemed to be interacting with the W51 main molecular cloud. A possible scenario for the formation of the dense clump can be outlined based on the collision of the filament with W51 main (Kang et al. 2010). In this scenario, cloud-cloud collision lead to the compression of the interface region and initiate the formation of stars as seen in W51 (Habe \& Ohta 1992). The molecular clumps at the interface are heated, but the molecular clumps on the trailing side remain cold and hence appear in absorption. Models for collision between two different clouds suggest that such collisions disrupt the larger cloud while the small cloud is compressed and subsequently forms stars. It is possible that the dense clump detected in absorption is formed on the trailing side of the filament that interacts with the main molecular cloud. Based on its density and somewhat elevated temperatures $(\sim 30 \mathrm{~K})$, it is also possible that this cloud is also collapsing to eventually form stars. In the absence of any observational evidence, we currently do not consider the source to be an IRDC. We suggest that the nondetection of this star-forming clump in the continuum is due to its chance coincidence along the line of sight with the much stronger W51e 2 continuum source.

Higher spectral and angular resolution observations of the foreground gas responsible for the absorption dip at $\sim 70 \mathrm{~km} \mathrm{~s}^{-1}$ are needed to understand its spatial distribution. We also propose to explore a clearer detection of the protostar using mid-infrared observations of the strong spectral features of molecular ices toward this region.

Acknowledgements. We thank the anonymous referee for the suggestions that helped improve the clarity of the paper significantly. HIFI has been designed and built by a consortium of institutes and university departments from across Europe, Canada and the United States under the leadership of SRON Netherlands Institute for Space Research, Groningen, The Netherlands and with major contributions from Germany, France and the US. Consortium members are: Canada: CSA, U. Waterloo; France: CESR, LAB, LERMA, IRAM; Germany: KOSMA, MPIfR, MPS; Ireland, NUI Maynooth; Italy: ASI, IFSI-INAF, Osservatorio Astrofisico di Arcetri-INAF; Netherlands: SRON, TUD; Poland: CAMK, CBK; Spain: Observatorio Astronómico Nacional (IGN), Centro de Astrobiologá (CSIC-INTA). Sweden: Chalmers University of Technology - MC2, RSS \& GARD; Onsala Space Observatory; Swedish National Space Board, Stockholm University - Stockholm Observatory; Switzerland: ETH Zurich, FHNW; USA: Caltech, JPL, NHSC. G. Hassel gratefully acknowledges helpful discussions and rate information provided by Yuri Aikawa. C. Vastel is grateful to F. Lique for providing the $\mathrm{CN}$ collisional rates in the LAMDA database format. T. Harrison thanks the Summer Scholars grant from the Center for Undergraduate Research and Creative Activity (CURCA), Siena College. This paper has made extensive use of the SIMBAD database, operated at CDS, Strasbourg, France. This publication makes use of molecular line data from the Boston University-FCRAO Galactic Ring Survey (GRS). The GRS is a joint project of Boston University and Five College Radio Astronomy Observatory, funded by the National Science Foundation under grants AST-9800334, AST-0098562, AST-0100793, AST0228993, \& AST-0507657. This paper is partly based on observations obtained with the IRAM Plateau de Bure interferometer and $30 \mathrm{~m}$ telescope. We are grateful to the IRAM staff at Plateau de Bure, Grenoble for their support during the observations and data reductions. IRAM is supported by INSU/CNRS (France), MPG (Germany), and IGN (Spain). Part of the research was carried out at the Jet Propulsion Laboratory, California Institute of Technology, under a contract with the National Aeronautics and Space Administration.

\section{References}

Aikawa, Y., Wakelam, V., Hersant, F., Garrod, R. T., \& Herbst, E. 2012, ApJ, 760, 40

André, P., Men'shchikov, A., Bontemps, S., et al. 2010, A\&A, 518, L102

Arnal, E. M., \& Goss, W. M. 1985, A\&A, 145, 369

Bally, J., Lanber, W. D., Stark, A. A., \& Wilson, R. W. 1987, ApJ, 312, L45

Bergin, E. A., Hogerheijde, M. R., Brinch, C., et al. 2010, A\&A, 521, L33

Bieging, J. 1975, in H II Regions and Related Topics, eds. T. L. Wilson, \& D. Downes (Berlin: Springer), Lect. Notes Phys., 42, 443

Blake, G. A., Sutton, E. C., Masson, C. R., \& Phillips, T. G. 1987, ApJ, 315, 621 Brown, P. D., Charnley, S. B., \& Millar, T. J. 1988, MNRAS, 231, 40

Carpenter, J. M., \& Sanders, D. B. 1998, AJ, 116, 1856

Cazaux, S., Caselli, P., \& Spaans, M. 2011, ApJ, 741, L34

Cheung, A. C., Rank, D. M., Townes, C. H., Thornton, D. D., \& Welch, W. J. 1968, Phys. Rev. Lett., 21, 1701

Coutens, A., Vastel, C., Caux, E., et al. 2012, A\&A, 539, A132

Coutens, A., Vastel, C., Cazaux, S., et al. 2013a, A\&A, 553, A75

Coutens, A., Vastel, C., Cabrit, S., et al. 2013b, A\&A, 560, A39

Danby, G., Flower, D. R., Valiron, P., Schilke, P., \& Walmsley, C. M. 1988, MNRAS, 235, 229

de Graauw, T., Helmich, F. P., Phillips, T. G., et al. 2010, A\&A, 518, L6

Faure, A., Hily-Blant, P., Le Gal, R., Rist, C., \& Pineau des Forêts, G. 2013, ApJ, 770, L2 
Flagey, N., Goldsmith, P. F., Lis, D. C., et al. 2013, ApJ, 762, 11 Garrod, R. T., \& Herbst, E. 2006, A\&A, 457, 927

Garrod, R. T., Wakelam, V., \& Herbst, E. 2007, A\&A, 467, 1103

Gaume, R. A., Johnston, K. J., \& Wilson, T. L. 1993, ApJ, 417, 645

Gerin, M., Kaźmierczak, M., Jastrzebska, M., et al. 2011, A\&A, 525, A116

Godard, B., Falgarone, E., Gerin, M., Hily-Blant, P., \& de Luca, M. 2010, A\&A, 520, A 20

Habe, A., \& Ohta, K. 1992, PASJ, 44, 203

Hasegawa, T. I., Herbst, E., \& Leung, C. M. 1992, ApJS, 82, 167

Hassel, G. E., Herbst, E., \& Garrod, R. T. 2008, ApJ, 681, 1385

Hassel, G. E., Harada, N., \& Herbst, E. 2011, ApJ, 743, 182

Hily-Blant, P., Maret, S., Bacmann, A., et al. 2010, A\&A, 521, L52

Indriolo, N., Neufeld, D. A., Gerin, M., et al. 2012, ApJ, 758, 83

Jacq, T., Walmsley, C. M., Henkel, C., et al. 1990, A\&A, 228, 447

Kalugina, Y., Lique, F., \& Klos, J. 2012, MNRAS, 422, 812

Kang, M., Bieging, J. H., Kulesa, C. A., et al. 2010, ApJS, 190, 58

Koo, B.-C. 1997, ApJS, 108, 489

Lique, F. 2010, J. Chem. Phys. 132, 044311

Liu, F.-C., Parise, B., Kristensen, L., et al. 2011, A\&A, 527, A19

Low, F. J., Young, E., Beintema, D. A., et al. 1984, ApJ, 278, L19

Lucas, R., \& Liszt, H. S. 2000, A\&A, 358, 1069

McElroy, D., Walsh, C., Markwick, A. J., et al. 2013, A\&A, 550, A36

Mehringer, D. M. 1994, ApJS, 91, 713

Molinari, S., Swinyard, B., Bally, J., et al. 2010a, A\&A, 518, L100

Molinari, S., Abergel, A., Rivera-Ingraham, A., et al. 2010b, 38th COSPAR

Scientific Assembly, Bremen, Germany

Mookerjea, B., Giesen, T., Stutzki, J., et al. 2010, A\&A, 521, L13

Mookerjea, B., Hassel, G. E., Gerin, M., et al. 2012, A\&A, 546, A75

Mufson, S. L., \& Liszt, H. S. 1979, ApJ, 232, 451

Müller, H. S. P., Schlöder, F., Stutzki, J., \& Winnewisser, G. 2005, J. Mol. Struct., 742,215

Neill, J. L., Wang, S., Bergin, E. A., et al. 2013, ApJ, 770, 142
Osamura, Y., Roberts, H., \& Herbst, E. 2005, ApJ, 621, 348

Ostriker, J. 1964, ApJ, 140, 1056

Ott, S. 2010, Astronomical Data Analysis Software and Systems XIX, ASP Conf. Ser., 434, 139

Pardo, J. R., Cernicharo, J., Herpin, F., et al. 2001, ApJ, 562, 799

Persson, C. M., Olofsson, A. O. H., Koning, N., et al. 2007, A\&A, 476, 807

Persson, C. M., Black, J. H., Cernicharo, J., et al. 2010, A\&A, 521, L45

Persson, C. M., De Luca, M., Mookerjea, B., et al. 2012, A\&A, 543, A145

Pety, J. 2005, SF2A-2005: Semaine de l'Astrophysique Française, eds. F. Casoli, T. Contini, J. M. Hameury, \& L. Pagani (EDP Sciences), 721

Pety, J., Teyssier, D., Fossé, D., Gerin, M., et al. 2005, A\&A, 435, 885

Pickett, H. M., Poynter, R. L., Cohen, E. A., et al. 1998, J. Quant. Spectr. Rad. Transf., 60, 883

Pilbratt, G. L., Riedinger, J. R., Passvogel, T., et al. 2010, A\&A, 518, L1

Radi, P. P., Bunn, T. L., Kemper, P. R., Molchan, M. E., \& Bowers, M. T. 1988, J. Chem. Phys., 88, 2809

Roelfsema, P. R., Helmich, F. P., Teyssier, D., et al. 2012, A\&A, 537, A17

Roueff, E., Felenbok, P., Black, J. H., \& Gry, C. 2002, A\&A, 384, 629

Sato, M., Reid, M. J., Brunthaler, A., \& Menten, K. M. 2010, ApJ, 720, 1055

Scott, P. F. 1978, MNRAS, 183, 435

Stantcheva, T., \& Herbst, E. 2003, MNRAS, 340, 983

Teyssier, D., Fossé, D., Gerin, M., et al. 2004, A\&A, 417, 135

Ungerechts, H., \& Thaddeus, P. 1987, ApJS, 63, 645

van der Tak, F. F. S., Walmsley, C. M., Herpin, F., \& Ceccarelli, C. 2006, A\&A, 447,1011

Viti, S., Collings, M. P., Dever, J. W., et al. 2004, MNRAS, 354, 1141

Whittet, D. C. B., Bode, M. F., Longmore, A. J., et al. 1988, MNRAS, 233, 321

Wilson, T. L., \& Rood, R. 1994, ARA\&A, 32, 191

Wilson, T. L., Mezger, P. G., Gardner, F. F., \& Milne, D. K. 1970, Astrophys. Lett., 5, 99

Yu, S., Pearson, J. C., \& Drouin, B. J., et al., 2010, J. Chem. Phys., 133, 17

Zhang, Q., \& Ho, P. T. P. 1997, ApJ, 488, 241 